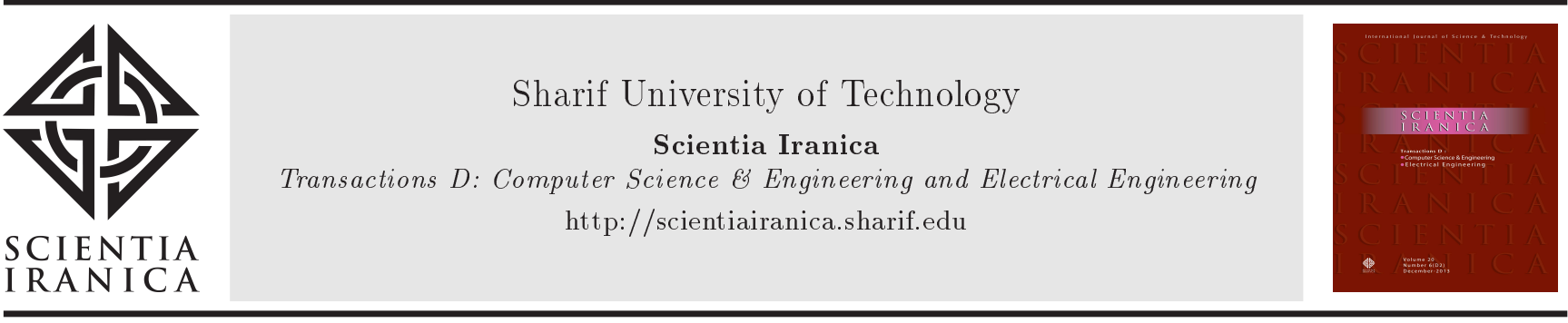

\title{
Control parameter-based comparative study of radiation properties of TMCCAA and TMHSCAA
}

\author{
G. Ram ${ }^{a, *}$, R. Kar ${ }^{b}$, and D. Mandal ${ }^{\mathrm{b}}$ \\ a. Department of Electronics and Communication Engineering, National Institute of Technology Warangal, India, Pin Code 506004. \\ b. Department of Electronics and Communication Engineering, National Institute of Technology Durgapur, India, Pin Code 713209. \\ Received 4 July 2019; received in revised form 6 July 2020; accepted 24 August 2020
}

\section{KEYWORDS \\ Collective Animal \\ Behavior (CAB); \\ Optimization \\ parameters; \\ Radiation pattern; \\ Time-modulated \\ antenna array.}

\begin{abstract}
Two different configurations were used in this study for the optimal design of the radiated electric field pattern characteristics of antenna arrays. In addition, the array patterns of the isotropic elements of two circular array geometries were studied. TimeModulated Concentric Circular Antenna Array (TMCCAA) and Time-Modulated Half Symmetric Circular Antenna Array (TMHSCAA) were also used for comparative analysis of the control parameters of the radiation properties of the antenna array. Collective Animal Behavior (CAB), an evolutionary approach, was then applied as the optimization tool. The effect of different control parameters of the TMCCAA radiation pattern was compared by considering two different cases, Case-1 and Case-2. Optimization of the spacing between each element in each ring indirectly led to the optimization of several elements in each ring. For TMCCAA, 279 isotropic elements, each containing one element at the centre, in an array were taken into account. In the second array, the impacts of different control parameters of the radiation pattern of TMHSCAA were compared by considering two different cases. Finally, the simulation results obtained for 20- element TMHSCAA with uniform amplitude and spacing were analyzed. Optimized SLL values obtained for Case-1 and Case- 2 of TMCCAA were obtained as $-24.68 \mathrm{~dB}$ and $-46.15 \mathrm{~dB}$, respectively.

(C) 2022 Sharif University of Technology. All rights reserved.
\end{abstract}

\section{Introduction}

The concept of array in antenna terminology has been developed to enhance the gain and directivity of any kind of antenna elements; yet, it is quite complicated, tedious, and costly to improve them at the individual element level. The main advantage of the antenna array is that one does not need to change the individual antenna dimensions [1]. It is an arrangement of an identical or non-identical antenna array as per the

\footnotetext{
*. Corresponding author. Tel.: +91-9679983382 E-mail addresses: gopi.ram@nitw.ac.in (G. Ram); rajibkarece@gmail.com (R.Kar); durbadal.bittu@gmail.com (D. Mandal)
}

doi: 10.24200 /sci. 2020.53945 .3503 requirement of the radiation characteristics. The arrays are found in different types of structures, namely linear, circular, rectangular, and hybrid geometry structures. The pattern measurement for the antenna array far-field electric field is done based on three parameters, namely the current excitation, spacing between the elements, and excitation phase. These three parameters bear an imperative responsibility and restrain the quality of the radiation from the antenna array structure. Of note, optimization of different control parameters faces different levels of complexity. For instance, optimization of the excitation phase is too complex and costly to implement. In addition, the optimization and implementation of the excitation amplitude and distance between two consecutive antennas are easier than optimizing the excitation phase. Apart from the three fundamental restraint criteria 
used for radiation quality enhancement from antenna theory, there is one more robust and emerging control parameter known as "switching time" [2]. This can be incorporated in any conventional technique due to its considerable flexibility to adjust time as a control parameter [3-5]. Sideband generated due to the Radio Frequency (RF) switch is reduced by the optimization techniques [6-9]. Different time sequences are opted for the optimization $[10,11]$. Directional null in the timemodulated array was also presented in [12].

Optimization of the antenna array [13] is a complex problem that is non-linear and non-differentiable. Therefore, finding solutions to different types of a complex problem is impossible by conventional optimization or numerical techniques. Circular and concentric circular array geometries were presented in [14-18]. To overcome this problem, the evolutionary algorithm is applied to work out the composite nonlinear and non-differentiable problems [19-26]. There are many evolutionary algorithms which are used for different fields of engineering [27-35] and optimization of antenna problem [36-40]. Development of element level antenna like the dielectric resonator antenna was reported in [41]. Apart from the optimization techniques, FTDT technique was also used in electromagnetics [42]. In this paper, Collective Animal Behavior (CAB)-based [43,44] optimization technique was used for the SLL reduction of Time-Modulated Concentric Circular Antenna Array (TMCCAA).

Section 2 discusses the theoretical background of TMCCAA. Section 3 presents and analyzes the simulation results. Section 4 gives the concluding remark of this study.

\section{Theoretical background}

Consider the geometry of nine-ring TMCCAA, where a total number of the concentric rings is nine. The mathematical modeling of this geometry in terms of the isotropic element is represented by Array Factor (AF), as given below [15]. Figure 1 shows the graphical representation of Concentric Circular Antenna Array (CCAA) structure.

$$
\begin{aligned}
A F(\theta, I)= & e^{j 2 \pi f_{0} t}\left\{1+\sum_{p=1}^{P} \sum_{i=1}^{N_{p}} I_{p} U_{p}(t)\right. \\
& \left.\exp \left[j k r_{p} \sin \theta \cos \left(\phi-\phi_{p i}\right)\right]\right\}
\end{aligned}
$$

where $I_{p}, N_{p}$, and $U_{p}(t)$ are the control parameters of the antenna array. The significance of $I_{p}, N_{p}$, and $U_{p}(t)$ is their ability to control the radiation pattern of the antenna array. In addition, $I_{p}$ denotes the current excitation amplitude value in the $p$ th ring. There are nine rings in TMCCAA. In this research, $I_{p}$ is kept

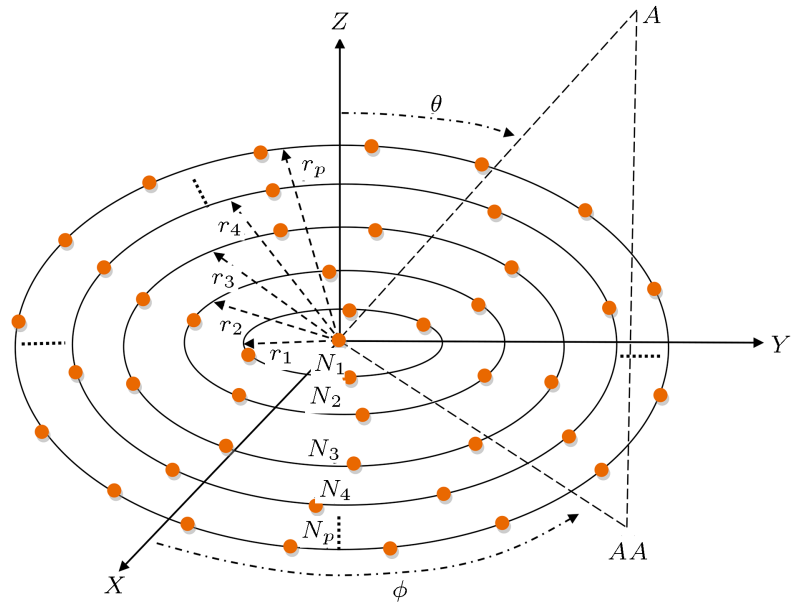

Figure 1. Graphical representation of Concentric Circular Antenna Array (CCAA) structure.

constant or uniform for the case study to avoid thecomplexity and the Dynamic Range Ratio (DRR) problem; $N_{p}$ denotes the total number of antennae present in the $p$ th ring; $r_{p}$ is the ring radius of the $p$ th ring; $\theta$ stands for the elevation plane on which the observation of the far-field is done; $f_{0}$ is the centre frequency; and $U_{p}(t)$ shows the switch connected with the ring. Due to the properties of the periodic RF switch in the $\mathrm{AF}$, it becomes time-modulated. Fourier series can carry out the AF frequency domain analysis of this periodic switch. Therefore, it will generate harmonic frequencies that correspond to the harmonic bands.

As a result, these harmonic frequencies will generate the harmonic pattern referred to as the sideband pattern. The first sideband corresponds to the first harmonic frequency; the second sideband corresponds to the second harmonic frequency, and so on. However, $95 \%$ of the sideband power is confined to the first five harmonic frequencies. Accordingly, Side-Band Level (SBL) in this paper is calculated until the 2nd harmonics.

Owing to the periodic nature of the switch, amplitude coefficients of the Fourier components can be represented as follows:

$$
a_{m p}=\frac{I_{p} \tau_{p}}{T_{p r f}} \sin c\left(m f_{p r f} \tau_{p}\right) e^{-j \pi m f_{p r f} \tau_{p}}
$$

where $m=0$ is for the fundamental frequency. Based on Eqs. (1) and (2), AF can be re-written in the frequency domain as Eq. (3) [15]:

$$
\begin{gathered}
A F_{m}(\theta, f)=\sum_{m=-\infty}^{\infty}\left\{1+\sum_{p=1}^{P} \sum_{i=1}^{N_{p}} a_{m p}\right. \\
\left.\exp \left[j k r_{p} \sin \theta \cos \left(\phi-\phi_{p i}\right)\right]\right\} \mathrm{e}^{j 2 \pi\left(f_{0}+m . f_{p r f}\right) t}
\end{gathered}
$$

Time-Modulated Half Symmetric Circular Antenna Array (TMHSCAA) was taken into account to make 


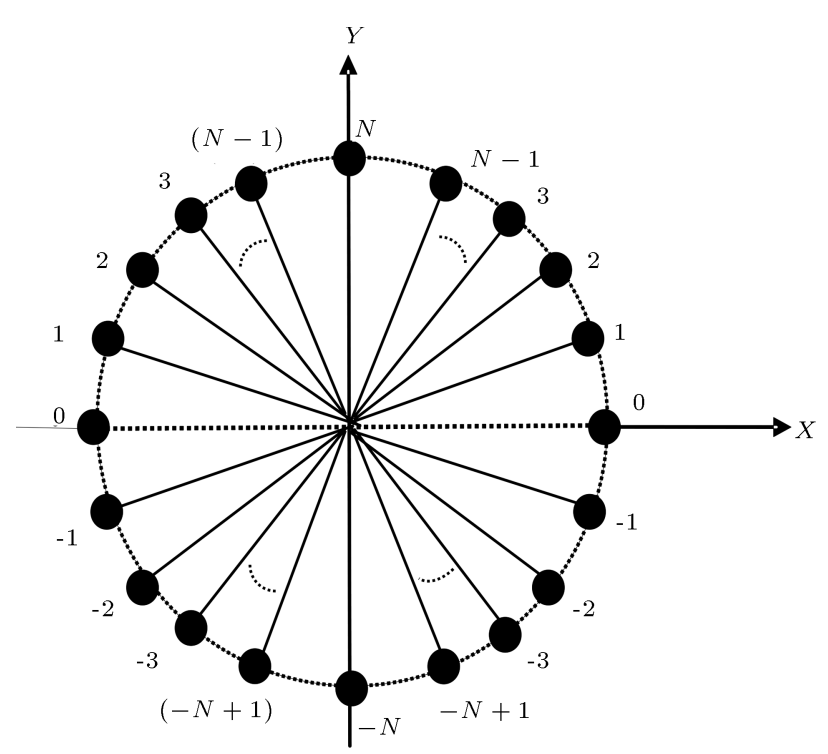

Figure 2. Graphical representation and its antenna arrangement of single-ring circular antenna array.

a comparison among the control parameters with $M$ $(=4 N)$ with uniform amplitude $I_{n}=I$, the phases $\alpha_{n}$, and switching sequences of $\tau_{n}[24,25]$. Figure 2 demonstrates a graphical representation and its antenna arrangement of the single ring circular antenna array.

$$
\begin{aligned}
A F(\phi, t)= & \sum_{m=-\infty}^{\infty} \sum_{n=-N}^{N} \gamma \mathrm{e}^{j 2 \pi\left(f_{0}+m . F_{p}\right) t} i_{m n} \cos \\
& {\left[k a \cos \left(\phi+\frac{2 n \pi}{M}\right)+\alpha_{n}\right] }
\end{aligned}
$$

where:

$$
\begin{aligned}
& \gamma=\left\{\begin{array}{l}
2 \text { for } n=(-N+1), \ldots-2 \ldots 0 \ldots 2 \ldots(N-1) \\
1 \quad \text { for } n=\{-N, N\}
\end{array}\right. \\
& i_{m n}=\frac{I_{n} \tau_{n}}{T_{p}} \sin c\left(\pi m F_{p} \tau_{n}\right) e^{-j \pi m F_{p} \tau_{n}} .
\end{aligned}
$$

Different harmonic components are present due to Furrier series decomposition represented by $f_{0}+m . F_{p}$, where $m$ shows the number of harmonic frequencies $(m=0, \pm 1, \pm 2, \ldots, \pm \infty)$. Therefore, sideband components $\left(f_{0}+m \cdot F_{p}, m \neq 0\right)$ are given by:

$$
\begin{aligned}
A F_{m}(\phi, t)= & \gamma \mathrm{e}^{j 2 \pi\left(f_{0}+m \cdot F_{p}\right) t} \sum_{n=-N}^{N} i_{m n} \cos \\
& {\left[k a \cos \left(\phi+\frac{2 n \pi}{M}\right)+\alpha_{n}\right] . }
\end{aligned}
$$

\section{Design of cost function}

The cost function for getting a better SLL pattern for TMCCAA is given below:

$$
\begin{aligned}
C F= & w_{1}^{*}\left[\sum_{-\frac{\pi}{2}}^{\theta_{L 1}} A F_{S L L}+\sum_{\theta_{L 2}}^{\frac{\pi}{2}} A F_{S L L}\right] \\
& +w_{2}^{*}\left[\sum_{\theta_{L 1}}^{\theta_{F N L}} A F_{S L L}+\sum_{\theta_{F N U}}^{\theta_{L 2}} A F_{S L L}\right] \\
& +w_{3}^{*} A F_{S B L_{1,2}}+w_{4}^{*} F N L .
\end{aligned}
$$

Eq. (7) represents the cost function for the objective of the paper. Here, $w_{1}=0.4, w_{2}=0.6, w_{3}=0.4$, and $w_{4}=1$ are the weighting factors assigned to different objectives of Eq. (7); $\theta_{F N L}$ and $\theta_{F N U}$ represent the lower and upper sides of the angular location of the first null of the main beam, respectively; $\theta_{L 1}$ and $\theta_{L 2}$ are the angular locations between $\left(-\pi / 2, \theta_{F N L}\right)$ and $\left(\theta_{F N U}, \pi / 2\right) ; A F_{S L L}$ is the calculated $\mathrm{AF}$ value on either side of the side lobe; and $A F_{S B L_{1,2}}$ denotes the calculated AF at the first and second harmonics, respectively.

The cost function for getting a better SLL pattern for TMHSCAA is given in the following.

$$
\begin{aligned}
C F= & w_{1} \times\left|A F_{S L L}\left(\phi_{1}, t\right)+A F_{S L L}\left(\phi_{2}, t\right)\right| \\
& /\left|A F_{M A X}(\phi, t)\right| \\
& +w_{2} \times\left(\prod_{\phi_{2}}^{\pi} A F(\phi, t)+\prod_{-\pi}^{\phi_{1}} A F(\phi, t)\right) \\
& +w_{3} \times\left[F N_{\text {current }}-F N_{\text {initial }}\right],
\end{aligned}
$$

where $\phi_{1}$ and $\phi_{2}$ are the azimuth angles at which null occurs on either sides of the main beam, and $A F_{M A X}(\phi, t)$ is the value of $\mathrm{AF}$ where the maximum main beam $A F(\phi, t)$ can be obtained. While the first term contributes to decreasing the SLL, the second one contributes to incorporation of nulls in the entire range of $\phi_{1}$ to $-\pi$ and $\phi_{2}$ to $\pi$. The third term maintains the computed First Null Beam Width (FNBW) and reference FNBW. Weighting factors $w_{1}, w_{2}$, and $w_{3}$ are set according to the priority of the optimization contribution, which is based on personal and group experience.

The cost function is taken into consideration to reduce the side lobe level of the radiation pattern. The primary objective of the cost function in this study was minimization problem. While the numerator represents the absolute value of the maximum side lobe, the denominator represents the maximum value of the main beam. This is ratio of maximum side lobe level to the main beam of the radiation pattern of the AF. When the variable parameter passes through the cost function with the help of $\mathrm{CAB}$, it will choose the appropriate value of the antenna variable parameter to be optimized. Therefore, once the number of iterations 
increases, $\mathrm{CAB}$ tries to minimize the cost function. The selected cost function is a minimization function, meaning that it tries to reduce the value of the side lobe level. The number of iterations can be fixed when there is no more improvement in or negligible tolerance for the cost function results.

\section{Results and discussion}

The mathematical expression for all array structures was coded and simulated in MATLAB platform. This section primarily presents the computational results for the designed nine-ring TMCCAA and 20-element TMHSCAA. The control parameters of the CAB algorithm are given in Table 1 . While considering the TMCCAA, let us consider the case where one opts only RF switches $\mathrm{ON}$ and $\mathrm{OFF}$ of each ring as the optimizing control parameters (Case-1). Another control parameter of the geometry is kept unchanged with respect to the un-optimized case of uniform 279element CCAA. The initial value of SLL was calculated as $-17.40 \mathrm{~dB}$ with a directivity value of $29.35 \mathrm{~dB}$. The current amplitude of each antenna element was also kept unchanged (constant) for both the cases. Followed by optimization of TMCCAA for Case- 1 using $\mathrm{CAB}$ algorithm, the radiation performance was significantly improved compared to that of the unoptimized case. SLL of $-24.68 \mathrm{~dB}$, SBL at the first harmonic of $-8.639 \mathrm{~dB}$, SBL at the second harmonic of $-15.4 \mathrm{~dB}$, FNBW of the degrees and directivity of 16.2 and $27.3038 \mathrm{~dB}$, respectively, were obtained using $\mathrm{CAB}$ algorithm. Figure 3 shows the obtained far-field electric field pattern of TMCCAA for Case-1. Now, consider another case where the RF switch, ring radii, and optimal distance between two consecutive antennas were regarded as the optimizing variables for optimization (Case-2). The geometry dimension depends on the optimized values of the ring radii as well as the distance between two consecutive antennas. Followed by the optimization of TMCCAA for Case- 2 using $\mathrm{CAB}$ algorithm, the radiation performance was considerably improved compared to that of both unoptimized case and Case- 1 .

SLL of $-46.15 \mathrm{~dB}$, sideband level at the first harmonic of $-5.733 \mathrm{~dB}$, sideband level at the second harmonic of $-10.63 \mathrm{~dB}$, FNBW of 15.4800 degree,

Table 1. Adjusting parameter of CAB.

\begin{tabular}{lc}
\hline Adjusting parameter & CAB \\
\hline Population size & 120 \\
No of fitness evolution & 500 \\
$B$ & 20 \\
$P$ & 0.5 \\
$H$ & 0.5 \\
\hline
\end{tabular}

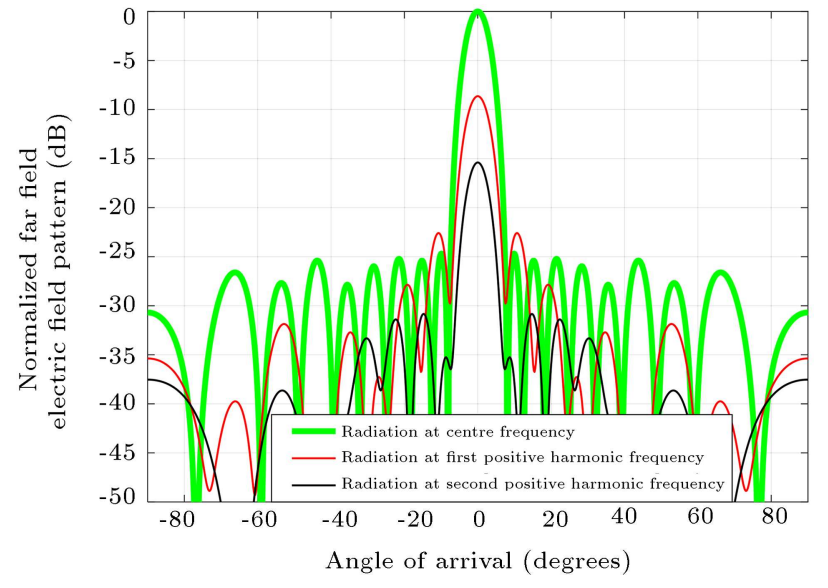

Figure 3. Obtained far-field electric field pattern of TMCCAA for Case-1 using CAB algorithm.

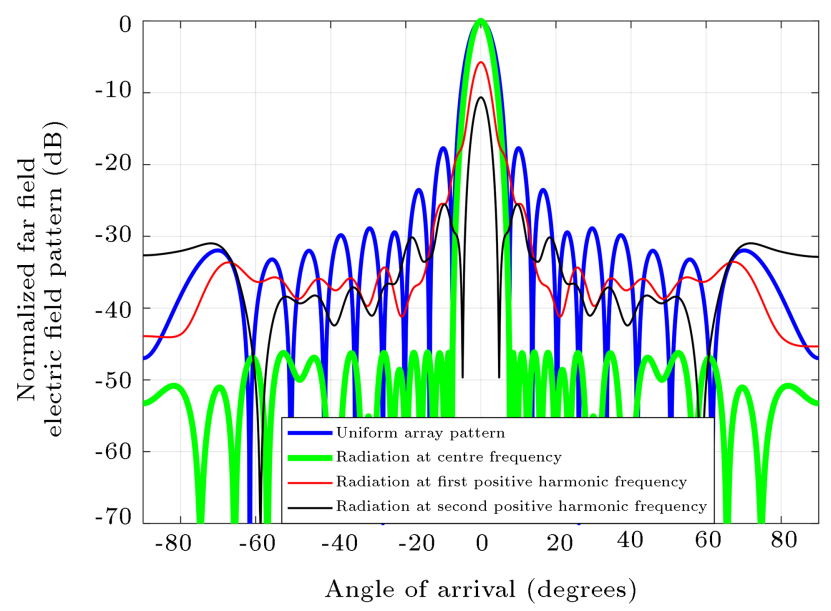

Figure 4. The obtained far-field electric field pattern of TMCCAA for Case-2 using CAB algorithm.

and directivity of 29.0770 were obtained for this case. The results for Case-2 obtained from CAB are also better than those reported in [24]. Figure 4 shows the obtained far-field electric field pattern of TMCCAA for Case-2. The FNBW obtained in Case-2 is approximately the same with that of the un-optimized case and Case- 1 .

Figure 5 makes a comparison of switch-on time sequences for Case- 1 and Case-2. Figure 6 also makes a comparison of the total numbers of antennae present in each ring of the CCAA for Case- 1 and Case-2. Figure 7 compares the ring radii of the CCAA for Case- 1 and Case-2.

Here, 20-element TMHSCAA RF-switch (ONOFF time sequence), connected to each antenna in the array, is acting as control parameter to discover the optimal far-field electric field pattern, as illustrated in Figure 8, where only RF switching is optimized. According to this figure, the measured values of SLL and FNBW obtained by CAB for 20-element TMHSCAA are $-9.36 \mathrm{~dB}$ and 60.48 degrees, respectively. Figure 9 


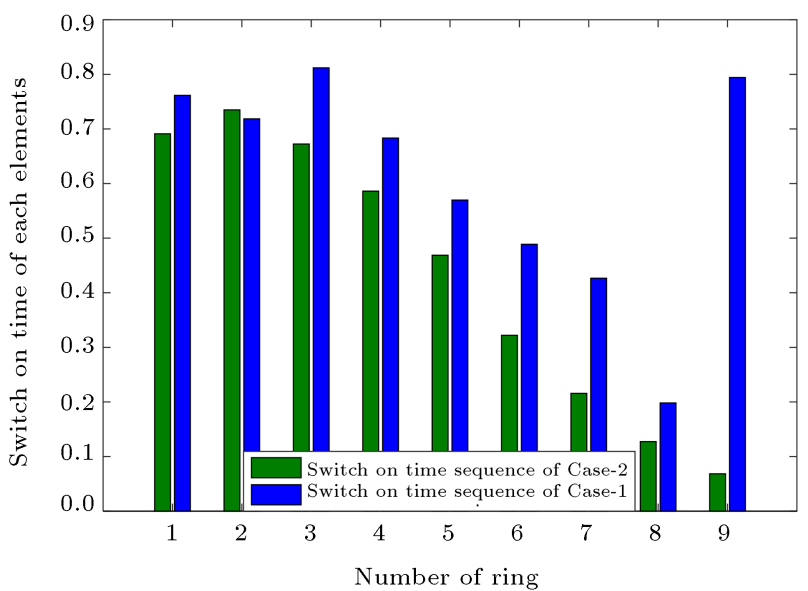

Figure 5. Comparison of switch-on time sequences for Case-1 and Case-2.

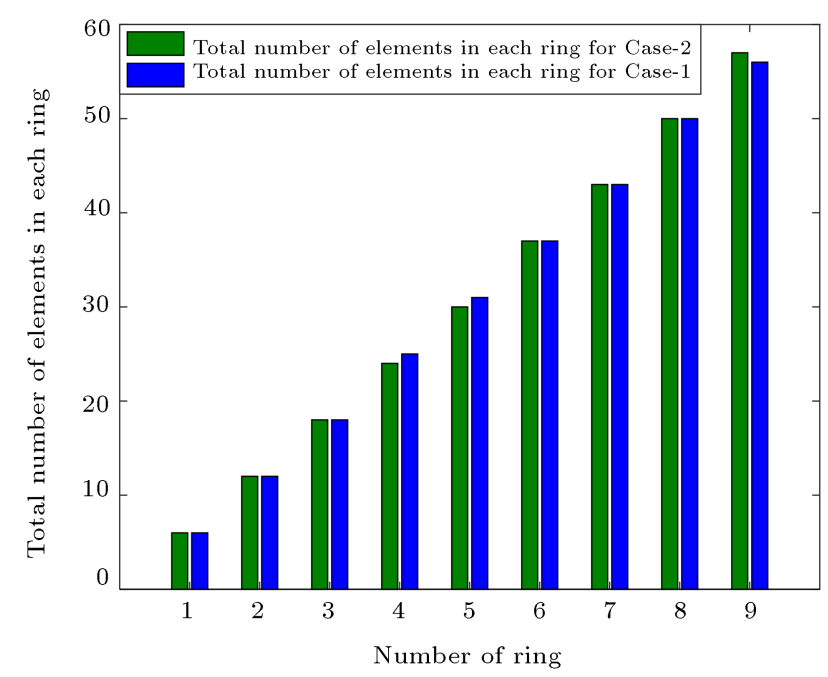

Figure 6. Comparison among the number of elements present in each ring of the concentric circular antenna array for Case-1 and Case-2.

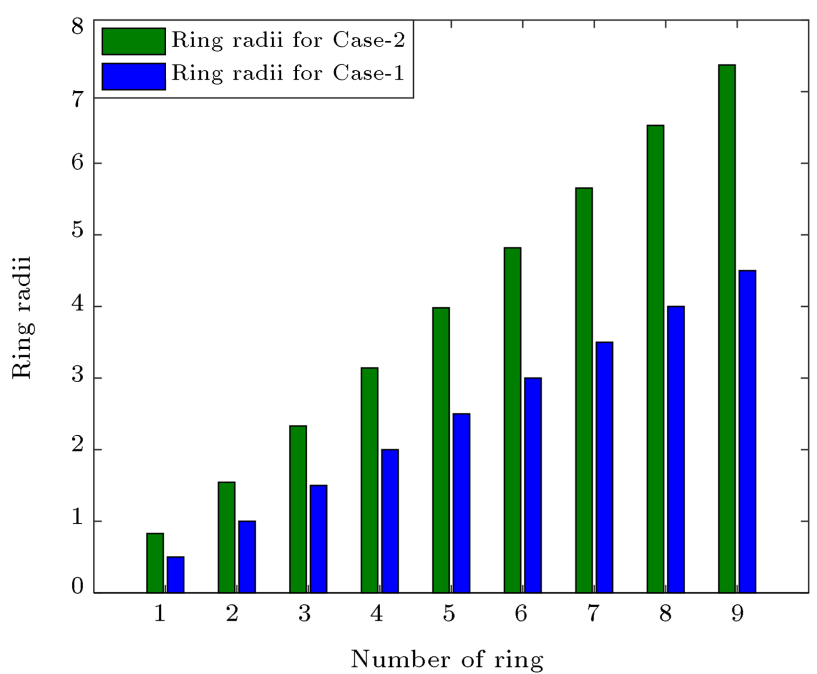

Figure 7. Comparison among the ring radii of the concentric circular antenna array for Case-1 and Case-2. presents the optimal values of the RF switching obtained for 20-element TMHSCAA.

Now, 20-element TMHSCAA RF switching and excitation phase of each antenna are the control parameters utilized to find the optimal far-field electric field pattern, as given in Figure 10, where RF switching and excitation phase of each antenna are optimized.

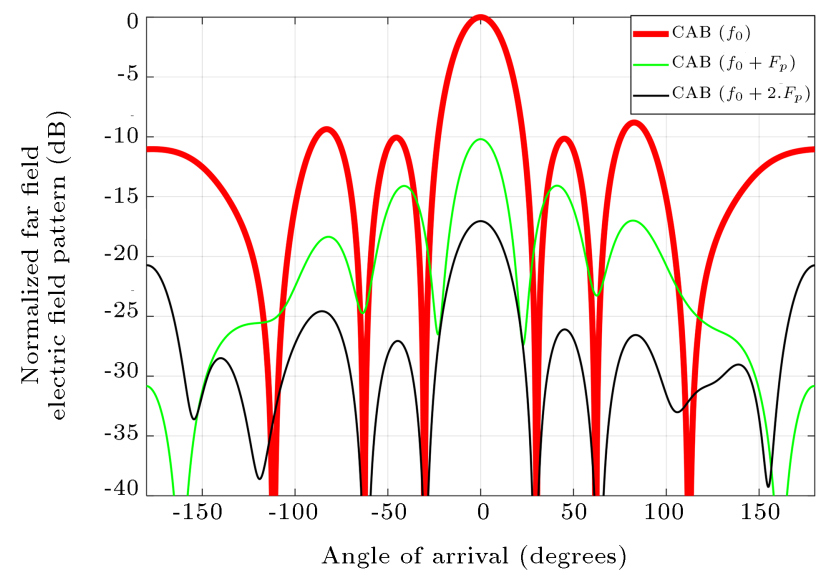

Figure 8. Obtained far-field electric field pattern of 20-elements TMHSCAA when only RF switching is optimized.

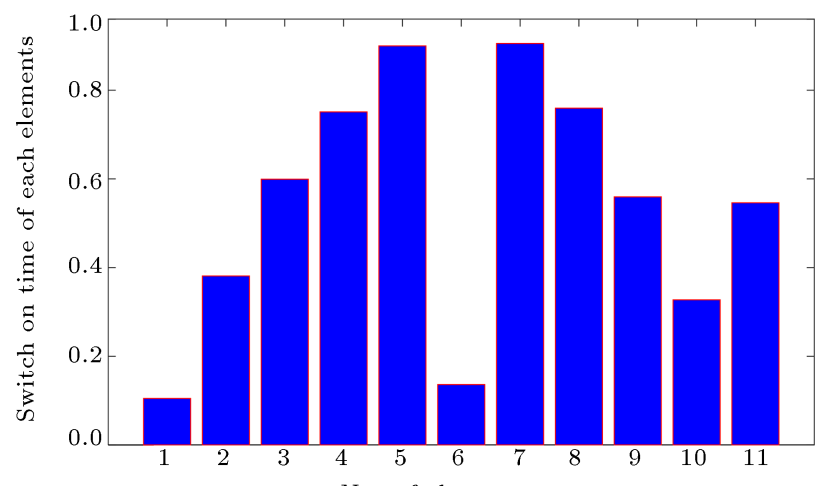

No. of elements

Figure 9. Optimal values of the RF switching obtained for 20-element TMHSCAA.

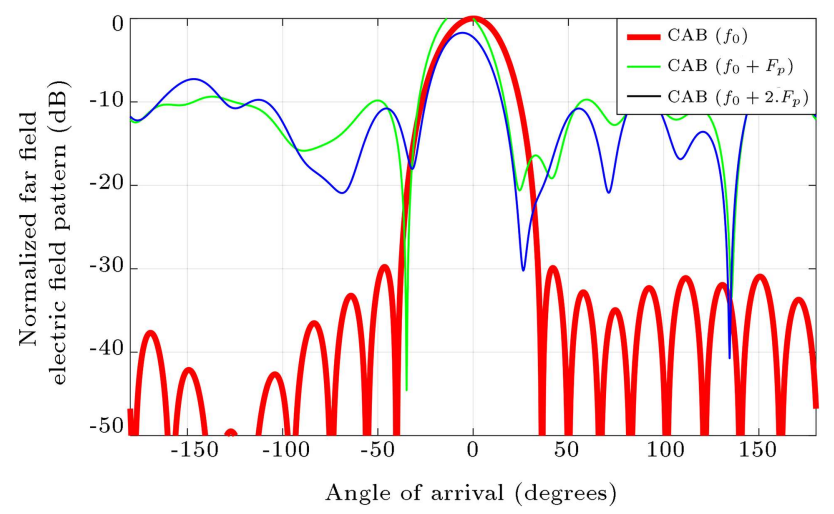

Figure 10. Obtained far-field electric field pattern of 20-element TMHSCAA when RF switching and excitation phase of each antenna are optimized simultaneously. 


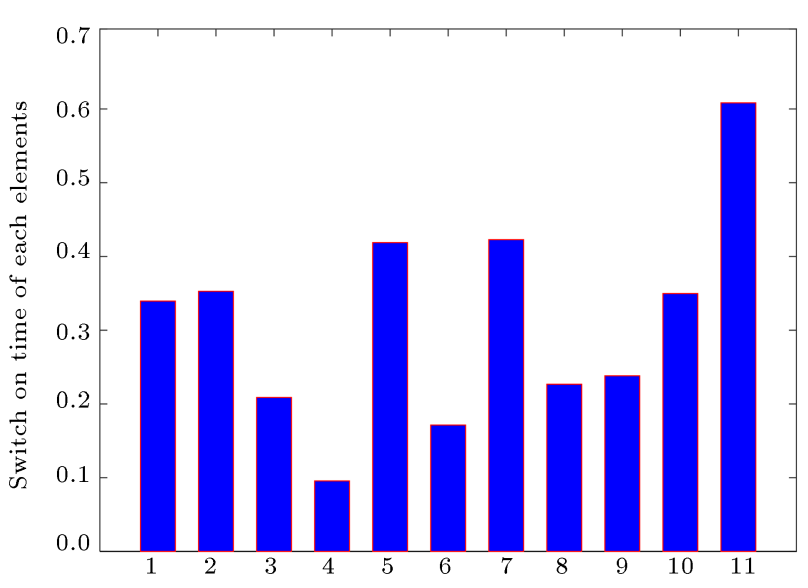

No. of elements

Figure 11. Optimal values of the RF switching obtained for 20-elements TMHSCAA when RF switching and excitation phase of each antenna are optimized simultaneously.

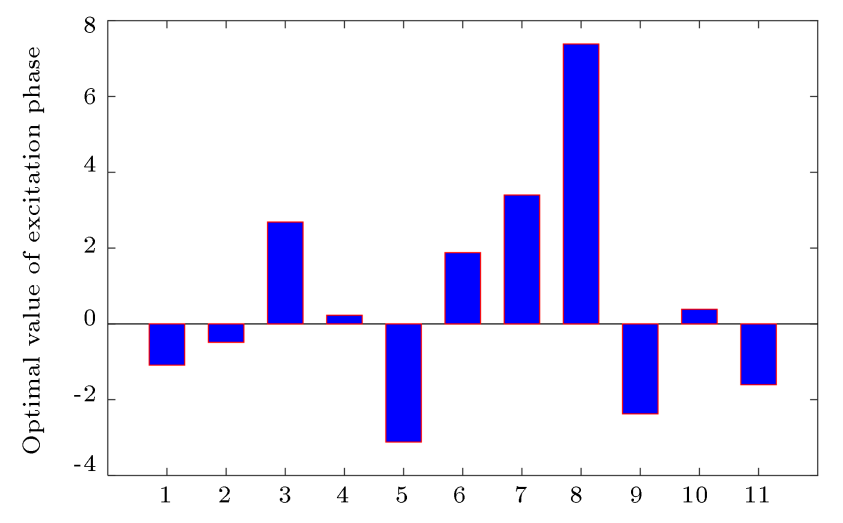

No. of elements needed to be optimized for excitation phase

Figure 12. Optimal values of the excitation phase of each element for 20-element TMHSCAA when RF switching and excitation phase of each antenna are optimized simultaneously.

According to the optimal far-field electric field pattern radiation shown in Figure 10, the measured values of SLL and FNBW obtained by CAB for 20element TMHSCAA were $-29.75 \mathrm{~dB}$ and 76.68 degrees, respectively. The obtained results for Case- 2 using CAB are substantially better than those reported in $[24,25]$. Figure 11 shows the optimal values of the RF switching obtained for 20-element TMHSCAA when RF switching and excitation phase of each antenna are optimized simultaneously. Figure 12 shows the optimal values of the excitation phase of each element for 20element TMHSCAA when RF switching and excitation phase of each antenna are optimized simultaneously.

All the computational results presented in this study facilitate decision-making, indicating that selection of the control parameters plays an important role in the performance enhancement of the radiation properties of any antenna array. However, the compu-

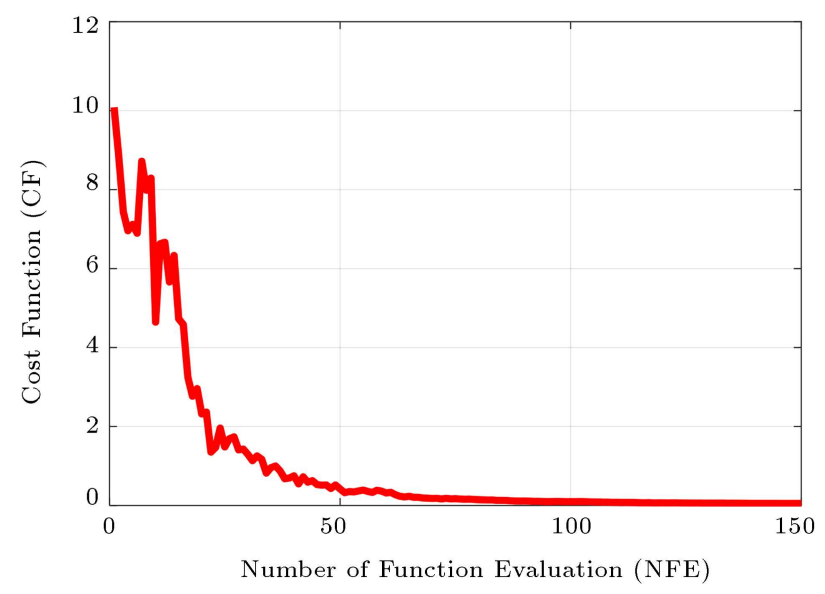

Figure 13. Convergence profile for obtained far-field electric field pattern of TMCCAA for Case-2 obtained using $\mathrm{CAB}$ algorithm.

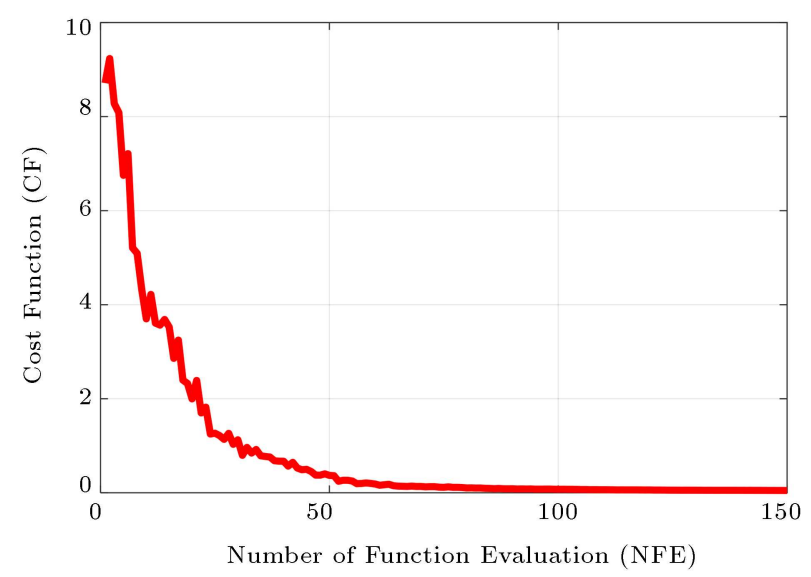

Figure 14. Convergence profile for 20-element TMHSCAA when RF switching and excitation phase of each antenna are optimized simultaneously obtained by CAB.

tational time will increase upon increasing the number of optimization variables.

Best convergence profiles for CAB independently running for fifty times are reported in Figures 13 and 14 according to which the $\mathrm{CAB}$ converges well to this particular optimization problem with 150 iterations performed for two different types of antenna array configurations. All computations were performed in MATLAB 9.5 on Core i5 (8th Gen) processor, $3 \mathrm{GHz}$ with 6 GB RAM.

\section{Conclusion}

Two comparative studies were performed, considering the optimizing variable parameters of the TimeModulated Concentric Circular Antenna Array (TMCCAA) and 20-element Time-Modulated Half Symmetric Circular Antenna Arrays (TMHSCAA), to compare the far-field electric field patterns. An evolutionary 
algorithm called Collective Animal Behavior (CAB) was held responsible for optimizing the SLL. Coding was implemented in the MATLAB platform. Based on the computational analysis, it could be concluded that the optimized results from the control parameters of Case- 2 were superior to those obtained by the control parameters of Case-1 as well as the un-optimized case. Optimized SLL obtained for the Case-1 of TMCCAA was $-24.68 \mathrm{~dB}$, whereas the optimized SLL obtained for Case- 2 was $-46.15 \mathrm{~dB}$. Thus, there was an improvement of $21.47 \mathrm{~dB}$ by switching from Case- 1 to Case-2. Therefore, it is clear that the selection of control parameters, cost function, and the selection of optimization algorithm play the most important role.

The 20-element TMHSCAA was taken as an example to compare the control parameters of the radiation pattern to obtain the lower SLL pattern to lower the SLL patterns, over those of the conventional arrays and other arrays designed. The numerical and statistical results show that $\mathrm{CAB}$ is capable of converging to much superior result.

\section{References}

1. Ballanis, C.A., Antenna Theory Analysis and Design, 3rd Edition, John Willey and Son's Inc., New York (2005).

2. Shanks, H.E. and Bickmore, R.W. "Four-dimensional electromagnetic radiators", Canad. J. Phys., 37, pp. 263-275 (1959).

3. Kummer, W.H., Villeneuve, A.T, Fong, T.S, et al. "Ultra-low side lobes from time-modulated arrays", IEEE Trans. Antennas Propagation., 11(5), pp. 633639 (1963).

4. Hardel, G.R., Yalapragada, N.T., Mandal, D., et al. "Introducing dipper nulls in time modulated linear symmetric antenna array using real coded genetic algorithms", IEEE Symposium on Computers and Informatics, pp. 249-254 (2011).

5. Yang, S., Gan, Y.B., and Tan, P.K. "Linear antenna arrays with bidirectional phase centre motion", IEEE Trans. Antennas Propagat., 53(5), pp. 1829-1835 (2005).

6. Yang, S., Gan, Y.B., and Qing, A. "Sideband suppression in time-modulated linear arrays by the differential evolution algorithm", IEEE Antennas Wireless Propag. Lett., 1, pp. 173-175 (2002).

7. Yang, S., Gan, Y.B., and Tan, P.K. "A new technique for power-pattern synthesis in time-modulated linear arrays", IEEE Antennas Wireless Propag. Lett., 2, pp. 285-287 (2003).

8. Fondevila, J., Brégains, J.C., Ares, F., et al. "Optimising uniformly excited linear arrays through time modulation", IEEE Antennas Wireless Propag. Lett., 3, pp. 298-301 (2004).
9. Yang, S., Gan, Y.B., Qing, A., et al. "Design of a uniform amplitude time modulated linear array with optimised time sequences", IEEE Trans. Antennas Propag., 53(7), pp. 2337-2339 (2005).

10. Yang, S., Gan, Y.B., and Tan, P.K. "Comparative study of low side lobe time modulated linear arrays with different time schemes", J. Electromagn. Waves Appl., 18(11), pp. 1443-1458 (2004).

11. Yang, S., Gan, Y.B., and Qing, A. "Moving phase centre antenna arrays with optimised static excitations", Microw. Opt. Tech. Lett., 38, pp. 83-85 (2003).

12. Ram, G., Chakravorty, P., Mandal, D., et al. "PSOIIW approach for nulling by RF switch in time modulated linear antenna arrays", International Conference on Electronics and Communication Systems (ICECS'15), Chennai, India, pp. 26-27 (2015).

13. Ram, G., Mandal, D., Kar, R., et al. "Optimised hyper beamforming of linear antenna arrays using firefly algorithm", International Journal of Microwave and Wireless Technologies, 6(2), pp. 181-194 (2014).

14. Ram, G., Mandal, D., Kar, R., et al. "Cat swarm optimization as applied to time-modulated concentric circular antenna array: analysis and comparison with other stochastic optimisation methods", IEEE Transactions on Antennas and Propagation, 63(9), pp. 4180-4183 (2015).

15. Ram, G., Mandal, D., Kar, R., et al. "Circular and concentric circular antenna array synthesis using cat swarm optimization", IETE Technical Review, 32(3), pp. 204-217 (2015).

16. Ram, G., Mandal, D., Kar, R., et al. "Pencil beam pattern synthesis of time-modulated concentric circular antenna array using PSO with Aging Leader and Challenger", Journal of Electromagnetic Waves and Applications, 29(12), pp. 1610-1629 (2015).

17. Krous, J.D., Antenna, McGraw Hill, New York (1950).

18. Mailloux, R.J. "Phased array architecture for millimetric active arrays", IEEE Antennas and Propagation Society Newsletter, 28(1), pp. 4-7 (1986).

19. Kaur, J., Khanna, R., and Kartikeyan, M.V. "Optimisation and development of O-shaped triple-band microstrip patch antenna for wireless communication applications", IETE Journal of Research, 60(2), pp. 95-105 (2014).

20. Yekan, S.T., Sadeghzadeh, R.A., and Moghadasi, N.M. "Microstrip-fed circularly polarised antenna array using semi-fractal cells for the implicational band", IETE Journal of Research, 60(6), pp. 383-388 (2014).

21. Ram, G., Mandal, D., Kar, R., et al. "CRPSOWM for linear antenna arrays with improved SLL and directivity", IETE Journal of Research, 61(2), pp. 109-120 (2015).

22. Kumar, V., Chhabra, J.K., and Kumar, D. "Automatic unsupervised feature selection using gravitational search algorithm", IETE Journal of Research, 61(1), pp. 22-31 (2015). 
23. Chandrasekaran, K., Simon, S.P., and Padhy, N.P. "Cuckoo search algorithm for emission reliable economic multi-objective dispatch problem", IETE Journal of Research, 60(2), pp. 128-138 (2014).

24. Ram, G., Mandal, D., Kar, R., et al. "Optimisation of radiation characteristic of time modulated circular geometry using DEWM", Scientia Iranica, Transactions D: Computer Science \& Engineering and Electrical Engineering, 25(3), pp. 71-1581 (2018).

25. Ram, G., Mandal, D., Kar, R., et al. "Radiation performance characteristic optimization of time-modulated circular antenna arrays", IETE Technical Review, 35(2), pp. 190-204 (2018).

26. Ram, G., Mandal, D., Kar, R., et al. "Opposition based gravitational search algorithm for synthesis circular and concentric circular antenna arrays", Scientia Iranica, Transactions D: Computer Science \& Engineering and Electrical Engineering, 22(6), pp. 24572471 (2015).

27. Ostadi, B., Sedeh, O.M., Kashan, A.H., et al. "An intelligent model to predict the day-ahead deregulated market clearing price: a hybrid NN, PSO and GA approach", Scientia Iranica, Transactions D: Computer Science \& Engineering and Electrical Engineering, 26(6), pp. 3846-3856 (2018).

28. Askari, E.A., Bashiri, M., and Moghaddam, R.T. "A capacitated bike-sharing location-allocation problem under demand uncertainty using sample average approximation: A greedy Genetic-Particle Swarm Optimization algorithm", Scientia Iranica, Transactions D: Computer Science \& Engineering and Electrical Engineering, 24(5), pp. 2567-2580 (2017).

29. Bhunia, A.K., Shaikh, A.A., Dhaka, V., et al. "An application of genetic algorithm and pso in an inventory model for single deteriorating item with variable demand dependent on marketing strategy and displayed stock level", Scientia Iranica, Transactions D: Computer Science \& Engineering and Electrical Engineering, 25(3), pp. 1641-1655 (2018).

30. Kaveh, A.A. and Nasrollahi, A. "Charged system search and particle swarm optimisation hybridised for optimal design of engineering structures", Sci. Iran. Trans. A, Civil Eng., 21(2), p. 295 (2014).

31. Manafifard, M., Ebadi, H., and Moghaddam, H.A. "Discrete particle swarm optimization for player trajectory extraction in soccer broadcast videos", Scientia Iranica, Transactions D: Computer Science \& Engineering and Electrical Engineering, 22(3), pp. 10311044 (2015).

32. Kaveh, A., Kaveh. A., and Shokohi, F. "A hybrid optimisation algorithm for the optimal design of laterallysupported castellated beams", Scientia Iranica, Transactions D: Computer Science \& Engineering and Electrical Engineering, 23(2), pp. 508-519 (2016).

33. Luo, Z., He, X., Chen, X., et al. "Synthesis of thinned concentric circular antenna arrays using modified TLBO algorithm", International Journal of Antennas and Propagation, 2015, p. 9 pages, Article ID 586345 (2015).

34. Bera, R., Kundu, K., and Pathak, N.N. "Optimal pattern synthesis of thinned and non-uniformly excited concentric circular array antennas using hybrid GSAPSO technique", Radio Engineering, 28(2), pp. 369385 (2019).

35. Hamdi, B., Limam, S., and Aguili, T. "Uniform and concentric circular antenna arrays synthesis for smart antenna systems using artificial neural network algorithm", Progress In Electromagnetics Research B, 67, pp. 91-105 (2016).

36. Mangoud, M.A., Elragal1, H.M., and Alshara1, M.T. "Design of time modulated concentric circular and concentric hexagonal antenna array using hybrid enhanced particle swarm optimisation and differential evolution algorithm", IET Microwaves, Antennas \& Propagation, 8(9), pp. 657-665 (2013). DOI: 10.1049/iet-map

37. Zare, A.S. and Baghaiee, S. "Application of ant colony optimisation algorithm to pattern synthesis of uniform circular antenna array", Applied Computational Electromagnetics Society Journal, 30(8), pp. 810-818, ISSN: $1054-4887$ (2015).

38. Mallick, S., Kar, R., Mandal, D., et al. "Optimal sizing of CMOS analogue circuits using gravitational search algorithm with particle swarm optimisation", International Journal of Machine Learning and Cybernetics, 8(1), pp. 309-331 (2017). DOI: 10.1007/s13042-0140324-3

39. Das, A., Mandal, D., and Kar, R. "An optimal far-field radiation pattern synthesis of time-modulated linear and concentric circular antenna array", International Journal on Numerical Modelling, 32(6) (2019).

40. Babayigit, B. "Synthesis of concentric circular antenna arrays using dragonfly algorithm", International Journal of Electronics, 105(5), pp. 784-793 (2017).

41. Mukherjee, B., Patel, P., and Mukherjee, J. "Hemispherical dielectric resonator antenna based on apollonian gasket of circles-a fractal approach", IEEE Transactions on Antennas and Propagation, 62(1), pp. 40-47 (2014).

42. Mukherjee, B. and Kumar, D. "Application of finite difference time domain to calculate the transmission coefficient of an electromagnetic wave impinging perpendicularly on a dielectric interface with modified MUR-I ABC", Defence Science Journal, 62, pp. 228235 (2012).

43. Ram, G., Mandal, D., Kar, R., et al. "Optimised hyper beamforming of linear antenna arrays using collective animal behaviour", The Scientific World Journal, 2013, Article ID 982017, pp. 1-13 (2013).

44. Cuevas, E., Gonzalez, M., Zaldivar, D., et al. "An algorithm for global optimisation inspired by collective animal behaviour", Discrete Dynamics in Nature and Society, 2012, Article ID 638275, 24 pages (2012). 


\section{Appendix}

Collective Animal Behavior (CAB) [43,44] mimics the collective behaviours of the animals. This algorithm functions based on the interaction behavior of the collective animals. In the group of animals, each position represents the solution within the search space. The cost function value represents the dominant nature of the animal with respect to the group. The best solution (best animal position) can be obtained by storing and updating two different memory elements: one is the memory of the best location at each generation $\left(M_{g}\right)$ and the other is the memory of the best historic position after complete evaluation $\left(M_{h}\right)$. $\mathrm{CAB}$ follows the following operations to reach the termination criterion.

The first process generates the random population matrix based on the numbers of population and variable parameters to be optimized. The upper and lower boundaries of each variable parameter depend on their constraints. For example, the upper and lower boundaries for the current excitation amplitude are within the range of 0 to 1 . It can be of any arbitrary value between 0 and 1 . Similarly, the inter-element spacing between each antenna element can be of any value between $0.5 \lambda$ and $\lambda$. The current study assessed two geometries of the antenna array with different optimization parameters, as previously mentioned in the results section.

Consider the initial set of the population of vector $A$ which is represented by $\left(A=\left\{a_{1}, a_{2}, \ldots, a_{N_{p}}\right\}\right)$, where $N p$ is the number of animals. The position of each animal $a_{i}$ is represented by the $D$-dimensional vector, where $D$ is the number of variable parameters to be optimized by $\mathrm{CAB}$ algorithm

In general, the upper and lower limits can be expressed as [44]:

$$
\begin{aligned}
& a_{j, i}=a_{j}^{l o w}+\operatorname{rand}(0.1) \cdot\left(a_{j}^{h i g h}-a_{j}^{l o w}\right) \\
& j=1,2, \ldots, D ; \quad i=1,2, \ldots, N_{p} .
\end{aligned}
$$

where $a_{j, i}$ is the $j$ th parameter of the $i$ th individual.

Vector $A$ is sorted based on the cost function, and a new vector $X=\left\{X_{1}, X_{2}, \ldots, X_{N_{p}}\right\}$ is generated so that the best $B$ positions are chosen.

Store the best positions of each animal according to the following equation [44]:

$$
a_{l}=m_{h}^{l}+v
$$

where $l \in\{1,2, \ldots, B\}, m_{h}^{l}$ shows the l-element of the historical memory $M_{h}$, and $v$ represents the random vector of small length.

Movement of the animals from/to the nearby ones based on the local attraction and repulsion behavior can be represented as [44]: $a_{i}=$

$\left\{\begin{array}{l}X_{i} \pm r \cdot\left(m_{h}^{\text {nearest }}-X_{i}\right) \quad \text { with probability } H \\ X_{i} \pm r \cdot\left(m_{g}^{\text {nearest }}-X_{i}\right) \quad \text { with probability }(1-H)\end{array}\right.$

where $i \in\left\{B+1, B+2, \ldots, N_{p}\right\}$, and $m_{h}^{\text {nearest }}$ and $m_{g}^{\text {nearest }}$ represent the nearest elements of $M_{h}$ and $M_{g}$ to $X_{i}$, respectively.

Movement of the animals in a random manner is determined according to the following equation [44]:

$$
a_{i}= \begin{cases}r & \text { with probability } P \\ X_{i} & \text { with probability }(1-P)\end{cases}
$$

where $i \in\left\{B+1, B+2, \ldots, N_{p}\right\}$, and $r$ represents the random vector of small length within the search space.

Compare the obtained solution space considering the following procedure:

i. Elements of $M_{h}$ and $M_{g}$ are merged into $M_{U}$ $\left(M_{U}=M_{h} \cup M_{g}\right)$;

ii. Each element $m_{U}^{i}$ of the memory $m_{U}$ is compared pair-wise to the remaining memory elements $\left(\left\{m_{U}^{1}, m_{U}^{2}, \ldots, m_{U}^{2 B-1}\right\}\right)$ based on the cost function value and $B$ best values are selected to build the new $M_{h}$.

\section{Biographies}

Gopi Ram received BE degree in Electronics and Telecommunication Engineering from Government Engineering College, Jagdalpur, Chhattisgarh, India in 2007. He also received MTech and PhD degrees from National Institute of Technology, Durgapur, West Bengal, India 2011 and 2016, respectively. He received a Scholarship from the Ministry of Human Resource and Development (MHRD), Government of India in 2009-2011 (MTech) and 2012-2016 (PhD). He joined NIT Warangal in April 2018 as an Assistant Professor at the ECE Department. His research interests include analysis and synthesis of radiation pattern of time-modulated antenna array structures, evolutionary optimization techniques, application of soft computing in electromagnetics, RF and microwave, and soft computing techniques. He has published more than 50 research papers in the peer reviewed international journals and conferences.

Rajib Kar passed BE degree in Electronics and Communication Engineering from Regional Engineering College, Durgapur, West Bengal, India in 2001. He also received his $\mathrm{MTech}$ and $\mathrm{PhD}$ degrees from $\mathrm{Na}$ tional Institute of Technology, Durgapur, West Bengal, India in 2008 and 2011, respectively. Currently, he is cooperating with National Institute of Technology, 
Durgapur, West Bengal, India as an Assistant Professor at the Department of Electronics and Communication Engineering. His research interests include VLSI signal processing and filter optimisation via evolutionary computing techniques. He is also a senior member of IEEE. He has published more than 370 research papers in international journals and conferences.

Durbadal Mandal received BE degree in Electronics and Communication Engineering from Regional Engineering College, Durgapur, West Bengal, India in 1996. He received his MTech and $\mathrm{PhD}$ degrees from National Institute of Technology, Durgapur, West Bengal, India in 2008 and 2011, respectively. Presently, he is cooperating with National Institute of Technology, Durgapur, West Bengal, India as an Assistant Professor at the Department of Electronics and Communication Engineering. His research interests include array antenna design and filter optimization via evolutionary computing techniques. He has published more than 400 research papers in international journals and conferences. 